\title{
Nanostructured Manganese Dioxide Thin Films prepared by a Novel Self-Assembly Process
}

\author{
PANG SUH CEM*, WEE BOON HONG \& CHIN SUK FUN \\ Department of Chemistry, Faculty of Resource Science and Technology, Universiti Malaysia Sarawak, 94300 Kota \\ Samarahan, Sarawak, Malaysia
}

\begin{abstract}
We have reported herein a novel self-assembly horizontal submersion process for the deposition of nanostructured manganese dioxide thin films on metalized plastic supporting substrates at ambient temperature and pressure. Uniform manganese dioxide thin films were deposited directly onto metallized plastic supporting substrate via the spontaneous assembly of preformed manganese dioxide nanoparticles in the form of stable colloidal suspension. This process affords a facile approach for the deposition of manganese dioxide thin films by simply repeating the submersion process after the prior deposited layer had been air-dried completely. Thinfilm deposition process initially occurred through the spontaneous adsorption of manganese dioxide nanoparticles onto specific surface sites of the metalized substrate. Subsequent events of particle growth, clusters formation, and aggregation or self-organization of particle clusters eventually led to the deposition of nanostructured thin films which were nanoparticulate and highly porous in nature. The surface morphological characteristics of deposited thin films were observed to be significantly affected by the duration of submersion and the post-deposition calcination temperature. By modulating and optimizing these parameters, thin films of tailored microstructure could therefore be prepared. Optimized manganese dioxide thin films were observed to exhibit excellent capacitive behavior as evidenced by the almost perfectly rectangular shape of cyclic voltamograms within the potential range of 0.0 to $1.0 \mathrm{~V}$ (versus SCE) in mild aqueous $\mathrm{Na}_{2} \mathrm{SO}_{4}$ electrolyte. The cycling stability and reversibility of these films were evaluated by prolonged charge-discharge cycling and no substantial deterioration of performance in terms of charge capacity and capacitive behaviors were observed after 1000 cycles. We speculate that the high capacitance value exhibited by self-assembled manganese dioxide thin films in mild aqueous electrolyte could be attributed to reversible and homogenous intercalation and deintercalation of protons during the charge and discharge cycling. The potential utility of self-assembled manganese dioxide thin films for the fabrication of electrochemical devices, in particular thin-film electrochemical capacitors is therefore envisaged.
\end{abstract}

Keywords: Manganese dioxide, nanoparticles, self-assembly, thin films, electrochemical capacitors.

\section{INTRODUCTION}

The utility of manganese dioxide $\left(\mathrm{MnO}_{2}\right)$ as electrode material for the fabrication of primary and secondary electrochemical cells has long been established due to its favorable electrochemical characteristic and inexpensive cost of production $(\mathrm{Qu}, 2006$ and Yagi et al., 2002). In recent decades, intense research interest has been focused on the utilization of $\mathrm{MnO}_{2}$ as electrode material for the fabrication of pseudocapacitive electrochemical capacitors as it is considered toxicologically benign and cheap. Pang et al. (2000) and Chin et al. (2002) had demonstrated a specific capacitance

*Corresponding author: scpang@frst.unimas.my of about $700 \mathrm{~F} / \mathrm{g}$ at $50 \mathrm{mV} / \mathrm{s}$ for ultra-thin nanoparticulate manganese dioxide thin films prepared by the sol-gel process, thereby provided further impetus for intensified research on nanoparticulate manganese dioxide thin films. Recent studies have concurred that nanoparticulate manganese dioxide thin films possess favorable electrochemical properties and are promising electrode materials for the fabrication of high power and energy density electrochemical capacitors.

Various chemical routes for the preparation of manganese dioxide thin-film electrodes that are suitable for the fabrication of electrochemical capacitors have been developed recently. Dai et al. (2006) prepared 
$\mathrm{MnO}_{2}$ thin films by electrostatic spray deposition from $\mathrm{KMnO}_{4}$ solution and reported a specific capacitance of $163 \mathrm{~F} / \mathrm{g}$ at scan rate of $5 \mathrm{mV} / \mathrm{s}$ in $0.2 \mathrm{M} \mathrm{Na} \mathrm{SO}_{4}$. Nanostructured manganese dioxide that was prepared by the sonochemical method had achieved a specific capacitance of $344 \mathrm{~F} / \mathrm{g}$ at scan rate of $5 \mathrm{mV} / \mathrm{s}$ in $0.5 \mathrm{M} \mathrm{Na}_{2} \mathrm{SO}_{4}$ electrolyte (Zolfaghari et al., 2007). Cathodic electrodeposited $\mathrm{MnO}_{\mathrm{x}}$ was reported to exhibit a specific capacitance of $425 \mathrm{~F} / \mathrm{g}$ at scan rate of $10 \mathrm{mV} / \mathrm{s}$ in $0.25 \mathrm{M}$ $\mathrm{Na}_{2} \mathrm{SO}_{4}$ electrolyte (Nagarajan et al., 2006). Potentiodynamical anodic co-deposited manganese oxide/carbon composite electrode was reported to possess a specific capacitance of $410 \mathrm{~F} / \mathrm{g}$ at the scan rate of $10 \mathrm{mV} / \mathrm{s}$ in $1.0 \mathrm{M}$ $\mathrm{Na}_{2} \mathrm{SO}_{4}$ electrolyte (Liu et al., 2007).

The present paper focuses on the characterization of nanostructured $\mathrm{MnO}_{2}$ thin films on nickel coated PET substrate prepared by a novel self-assembly approach under controlled conditions. The effect of deposition parameters such as duration of submersion and post-deposition calcination temperature on the microstructure and electrochemical properties of $\mathrm{MnO}_{2}$ thin films were investigated.

\section{MATERIALS \& METHOD}

\section{Preparation of $\mathrm{MnO}_{2}$ sol}

All chemicals were of analytical grades and were used as obtained without further purification. Stable $\mathrm{MnO}_{2}$ sol was prepared according to method reported by Perez-Benito et al. (1996) based on the following reaction stoichiometry:

$8 \mathrm{MnO}_{4}^{-}+3 \mathrm{~S}_{2} \mathrm{O}_{3}{ }^{2-}+2 \mathrm{H}^{+} \rightarrow 8 \mathrm{MnO}_{2}+6$ $\mathrm{SO}_{4}{ }^{2-}+\mathrm{H}_{2} \mathrm{O}$

\section{Deposition of $\mathrm{MnO}_{2}$ Thin Films}

A thoroughly cleaned nickel-coated PET substrate (3M PP2900) of dimension $40 \mathrm{~mm} \mathrm{x}$ $44 \mathrm{~mm}$ were positioned perfectly horizontal and flat in a glass petri dish with the aid of adhesive tape. $\mathrm{MnO}_{2}$ sol was then poured slowly into the glass petri dish until the substrate was completely submersed. After the predetermined submersion duration, the sol was then drained out meticulously using a micropipette. The $\mathrm{MnO}_{2}$ thin film formed spontaneously on the PET substrate was then air-dried under controlled environment.
Nanostructured $\mathrm{MnO}_{2}$ thin films of varying thicknesses were prepared through layer-bylayer self-assembly by simply repeating the horizontal submersion coating process the desired number of times. The deposited $\mathrm{MnO}_{2}$ film was left to dry in air overnight prior to heat treatment in a tube furnace at $200{ }^{\circ} \mathrm{C}$ in air for an hour.

\section{Physical and Electrochemical characterization of $\mathrm{MnO}_{2}$ Films}

The electroactive material loading and homogeneity of $\mathrm{MnO}_{2}$ thin film was evaluated by atomic adsorption spectroscopy (AAS). The electrodes were dissolved in the $\mathrm{H}_{2} \mathrm{O}_{2} / \mathrm{HNO}_{3}$ mixture solution and the concentration of $\mathrm{Mn}$ per unit volume $\left(\mathrm{cm}^{3}\right)$ was determined using atomic absorption spectroscopy (AAS). The electroactive material loading of $\mathrm{MnO}_{2}$ thinfilm electrode (mass per unit area $\left(\mathrm{g} / \mathrm{cm}^{2}\right)$ ) was calculated based on the Mn concentration as determined using the formula weight of stoichiometric $\mathrm{MnO}_{2}$ of $86.94 \mathrm{~g} / \mathrm{mol}$.

The surface morphology of the $\mathrm{MnO}_{2}$ thin films was observed using a field emission scanning electron microscope (FESEM, LEO 1525), whereas their impedance and capacitive behaviors were investigated by cyclic voltammetry (CV) and electrochemical impedance spectroscopy (EIS) operated on a computer interfaced Frequency Response Analyzer (PARSTAT 2263). Cyclic voltammetry $(\mathrm{CV})$ studies were performed in $1.0 \mathrm{M} \mathrm{Na} \mathrm{Na}_{2} \mathrm{SO}_{4}$ aqueous electrolyte using a standard 3-electrode configuration with platinum foil as the counter electrode and Saturated Calomel Electrode (SCE) fitted with a Vycor frit as the reference electrode. The surface area of the working electrodes was fixed at $0.126 \mathrm{~cm}^{2}$ and a scan rate of $50 \mathrm{mV} / \mathrm{s}$ within potential window of $0-1.0 \mathrm{~V}$ (vs SCE) was used. The specific capacitance (SC) of the $\mathrm{MnO}_{2}$ thin films was calculated by dividing the integrated anodic current $\left(\mathrm{Q}_{\mathrm{a}}\right)$ with the mass of electroactive material on electrode $(m)$ and the width of potential scan window $(\Delta V)$ according to equation (1).

Specific Capacitance $(\mathrm{SC})=\frac{1}{m \Delta V} \int \mathrm{I} \delta \mathrm{t}$ 
Electrochemical impedance spectroscopy (EIS) measurements for $\mathrm{MnO}_{2}$ thin-film electrodes were performed within the frequency range of $1 \mathrm{MHz}$ to $10 \mathrm{mHz}$. The impedances at 100 $\mathrm{mHz}$ and $10 \mathrm{mHz}$ were used for capacitance (C) calculation according to equation (2).

$$
\text { Capacitance, } \mathrm{C}=-\frac{1}{\omega \mathrm{Z}_{\mathrm{img}}}
$$

where, $\mathrm{C}$ is the capacitance, $\omega(=2 \pi f)$ is the angular frequency and $\mathrm{Z}_{\mathrm{img}}$ is the imaginary part of the impedance. Specific capacitance (SC) was calculated based on the capacitance values divided by the mass of electroactive material on the electrode.

\section{RESULTS \& DISCUSSION}

\section{Characterization of $\mathrm{MnO}_{2}$ Thin Films}

\section{Field Emission Scanning Electron Microscopy}

Figure 1 shows SEM micrographs of $\mathrm{MnO}_{2}$ thin films deposited on the nickel- coated PET substrates by the novel self-assembly horizontal submersion process. All SEM micrographs showed that $\mathrm{MnO}_{2}$ thin films were nanoparticulate in nature with particle size ranged between 30 to $50 \mathrm{~nm}$. Considerably large variation in pores sizes ranging from macropores to micropores were visually discernible and hence indicative of the porous nature of these films. However, $\mathrm{MnO}_{2}$ thin films prepared by repeating the deposition process with different number of times (3-5x) showed slightly increased film density and uniformity (Figure 1(b) and (c)). We speculate that the $\mathrm{MnO}_{2}$ film deposition process was being catalyzed by active sites available on the surface of nickel-coated PET substrate. This film deposition process was essentially a spontaneous self-assembly process which involved initial adsorption of manganese dioxide nanoparticles onto surface active sites of the metallized supporting substrate, subsequent particle growth, clusters formation, and aggregation of clusters, and eventually led to the deposition of nanostructured $\mathrm{MnO}_{2}$ thin film on the supporting substrate.

Figure 2 shows SEM micrographs of various $\mathrm{MnO}_{2}$ thin films of different coatings before and after heat treatment at $200{ }^{\circ} \mathrm{C}$ in air for an hour. $\mathrm{MnO}_{2}$ films generally showed increase in their film density and notably reduced aggregate sizes upon heat treatment, with thinner films (1 coating) showed comparatively higher degree of densification than that of thicker films (5 coatings).

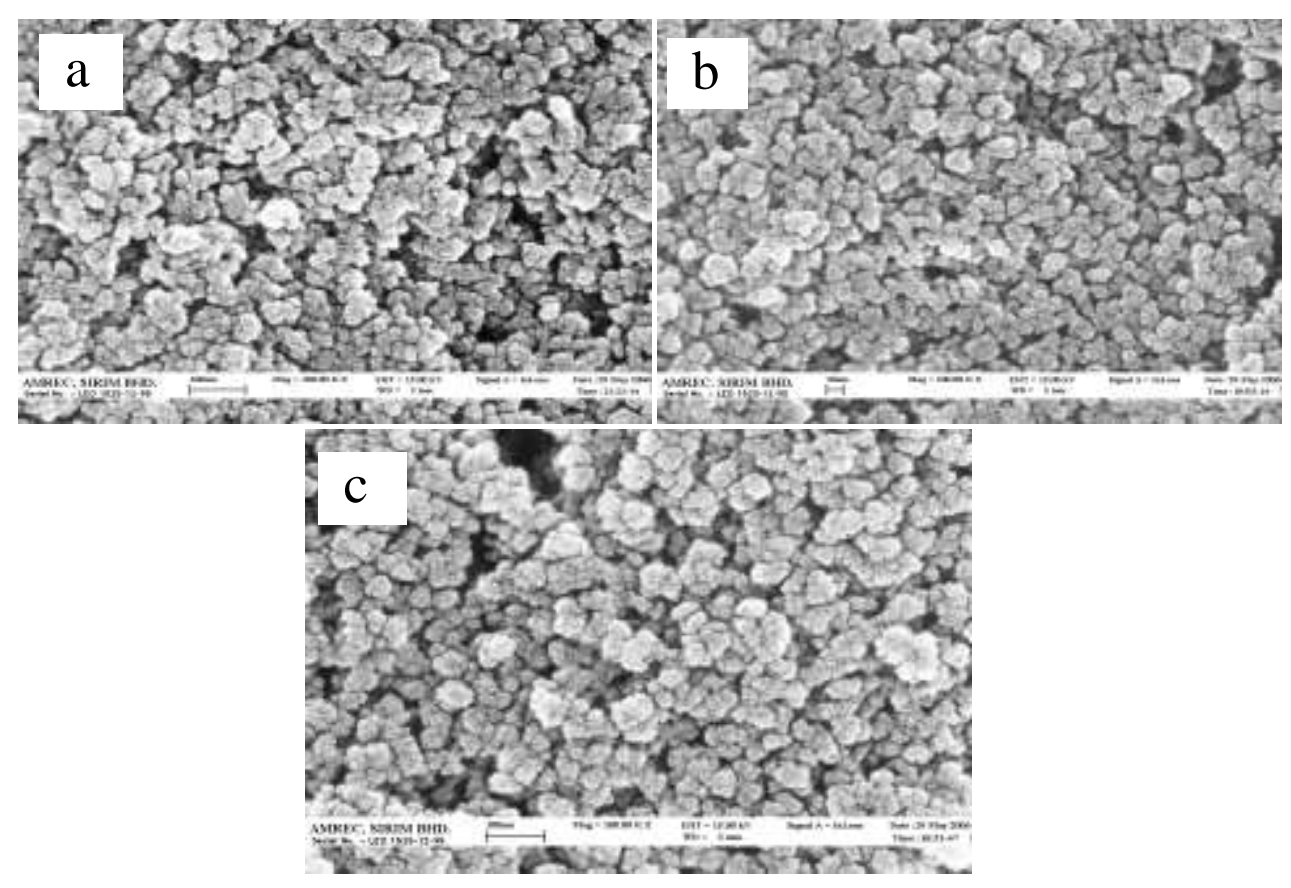

Figure 1. SEM micrographs of $\mathrm{MnO}_{2}$ films deposited on nickel-coated PET substrate prepared by repeating the self-assembly horizontal submersion process (a) 1x; (b) 3x, and (c) 5x. (Magnification: $100,000 \mathrm{x})$ 

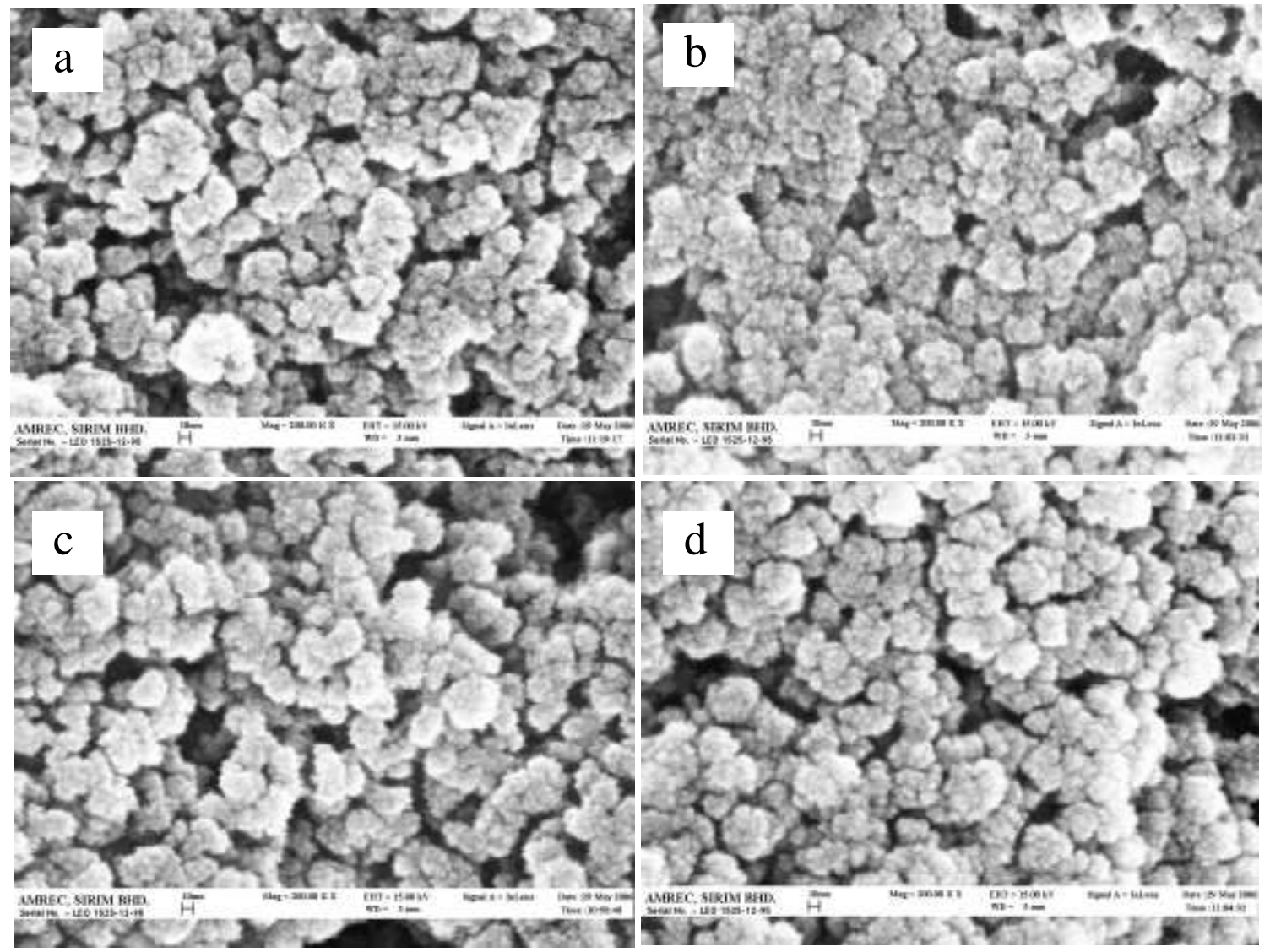

Figure 2. SEM micrographs of $\mathrm{MnO}_{2}$ thin films before and after heat treatment; (a) 1x before, (b) 1x after, (c) 5x before, and (d) 5x after (Magnification: 200,000 x).

All self-assembled $\mathrm{MnO}_{2}$ thin films showed good adhesion to the nickel-coated PET substrates even without the use of any binders. However, cracks were observed (at low magnification) on most film surfaces after heat treatment which could be attributed to film densification. Nonetheless, the highly porous nature of these films would facilitate facile accessibility of electrolyte ions into the bulk of the electrode materials during voltammetric cycling. The heat treatment could have effectively removed all physisorbed water and a substantial portion of chemisorbed water resulting in the densification of $\mathrm{MnO}_{2}$ thin films. The predominant advantage of film densification through heat treatment process was to afford enhanced electronic connectivity and conductivity between nanoclusters thereby contributed to substantial reduction of the internal resistance of films. Lower internal resistance of $\mathrm{MnO}_{2}$ films correlated directly to higher electrical conductivity, which in turn, augments their pseudocapacitive performance favorable for the fabrication of charge-storage devices. Besides, the presence of excess chemically bound structural water could perform a pivotal role in aiding the cationic/electrolyte accessibility in $\mathrm{MnO}_{2}$ films (Lee et al., 1999). Henceforth, an optimal balance between the effect of densification on reducing internal electrical resistance and the structural water content in the bulk electroactive material for promoting cationic diffusivity should be attained in order to achieve optimized pseudocapacitive performance.

The morphological structure of bulk electroactive material could substantially influence the accessibility of cations into internal surfaces of the electrode material. The smaller the pore sizes of the electroactive materials, the larger is its surface area and thence affords higher charge storage capacity. However, the pore size must be large enough for the intercalation of cations into the bulk of the electroactive material. Preparation of electroactive materials with desired pore size 
distribution has always been a very challenging task.

\section{Atomic Absorption Spectroscopy (AAS)}

In order to corroborate the homogeneity and uniformity of self-assembled $\mathrm{MnO}_{2}$ thin films, each selected thin film electrode was being cut into four smaller portions of same dimensions. These films were being dissolved in the $\mathrm{H}_{2} \mathrm{O}_{2} / \mathrm{HNO}_{3}$ mixture and the Mn concentrations were determined quantitatively using AAS. The total mean $\mathrm{MnO}_{2}$ loading of films were calculated to be $0.1029 \mathrm{mg} / \mathrm{cm}^{2}$ based on the concentration of $\mathrm{Mn}$ determined. Comparison between $\mathrm{MnO}_{2}$ loadings for these four smaller electrode portions showed good agreement which was deemed sufficient to substantiate the homogeneity and uniformity of deposited $\mathrm{MnO}_{2}$ thin films.

\section{Cyclic voltammetry $(C V)$}

Figure 3 shows the cyclic voltammograms of $\mathrm{MnO}_{2}$ thin-film electrodes with almost rectangular shape indicating good capacitive behaviors. The areas within these CV curves were observed to increase substantially with increased number of coatings by repeating the horizontal submersion process. This indicated that the charge capacity of deposited $\mathrm{MnO}_{2}$ films increased with their relative film thickness.

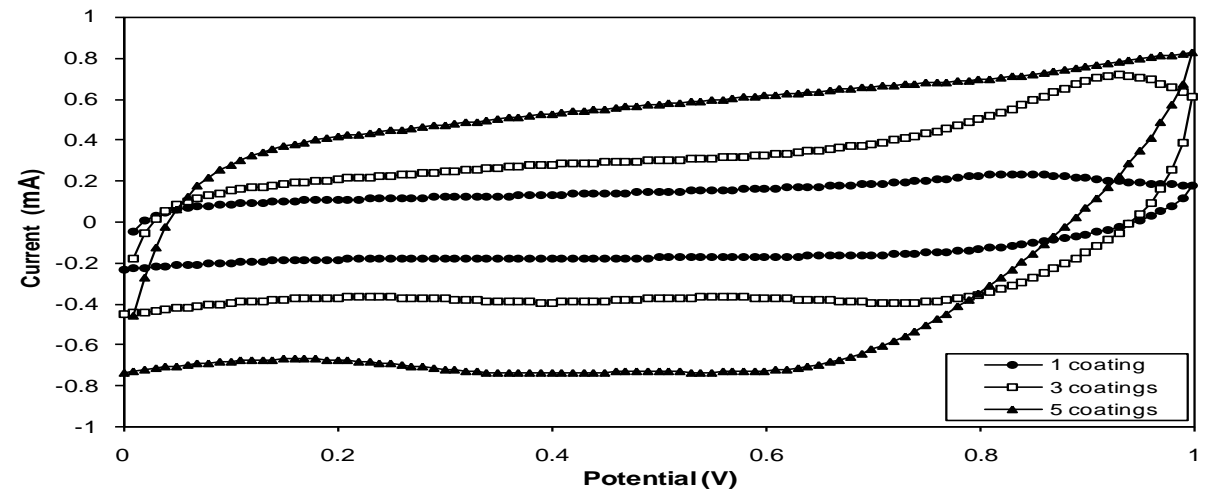

Figure 3. Cyclic voltammograms of $\mathrm{MnO}_{2}$ thin films prepared with different number of coatings and relative film thicknesses.

Figure 4 shows the effect of number of coatings on the charge capacity $\left(\mathrm{mF} / \mathrm{cm}^{2}\right)$ and $\mathrm{Q}_{\mathrm{a}} / \mathrm{Q}_{\mathrm{c}}$ ratio of $\mathrm{MnO}_{2}$ thin films. A positive linear correlation was observed between the charge capacity values and the number of coatings or relative film thicknesses. The $\mathrm{Q}_{\mathrm{a}} / \mathrm{Q}_{\mathrm{c}}$ ratios were approximately unity which indicated excellent reversibility of the charge/discharge processes that occurred within these $\mathrm{MnO}_{2}$ thin-film electrodes.

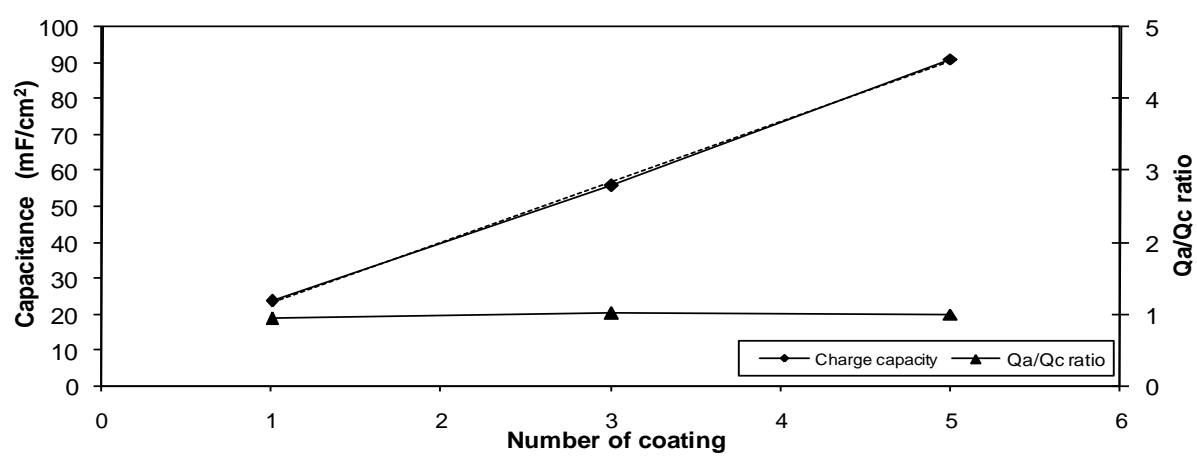

Figure 4. Effect of number of coatings on the electrochemical properties of self-assembled $\mathrm{MnO}_{2}$ thin films. 


\section{Performance Evaluation of Thin-film Electrochemical Capacitor Prototypes}

Cyclic voltammograms of electrochemical capacitor prototypes with different electrode configurations were being evaluated in $0.2 \mathrm{M}$ $\mathrm{Na}_{2} \mathrm{SO}_{4}$ liquid electrolyte and agar-based gel electrolyte containing dissolved $0.2 \mathrm{M} \mathrm{Na}_{2} \mathrm{SO}_{4}$ are as depicted in Figures $5-8$.
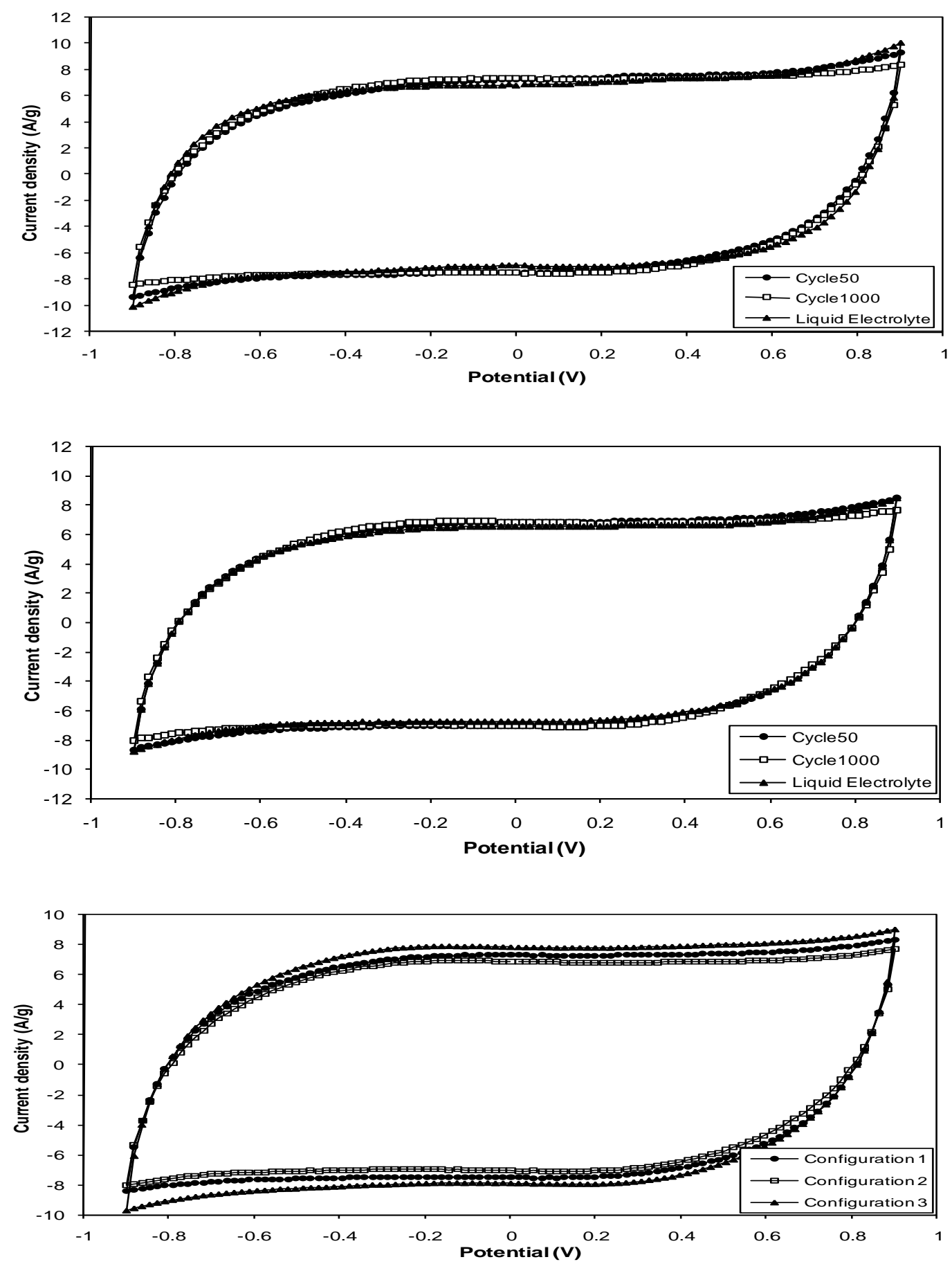

Figure 5. Cyclic voltammograms of prototype thin-film electrochemical capacitor with different electrode configurations, (a) Configuration 1, (b) Configuration 2, and (c) Comparison between electrode configuration $1,2 \& 3$. Cycles 50 and 1000 were obtained with all prototypes in agar-based gel electrolyte. 
All electrochemical capacitor prototypes with different device configurations were observed to exhibit excellent capacitive behaviors as evidenced by the almost perfectly rectangular shape of $\mathrm{CV}$ curves at the scan rate of $50 \mathrm{mV} / \mathrm{s}$ within the potential window of $-0.9 \mathrm{~V}$ to $0.9 \mathrm{~V}$. No redox peaks were discernible within these $\mathrm{CV}$ curves even after prolong cycling test of exceeding 1,000 cycles, which could be attributed to high phase stability and reversibility of the pseudocapacitive electrochemical reactions occurring within these prototype devices.

Figure 6 shows the cycling behaviors of electrochemical capacitor prototypes with different electrode configurations. The maximum specific capacitance achieved for all these prototypes ranged between $123 \mathrm{~F} / \mathrm{g}$ and $141 \mathrm{~F} / \mathrm{g}$. All prototypes exhibited excellent cycling stability without showing any loss in their capacitance, albeit an overall capacitance increment of about 2 to $4 \%$ upon cycling for 1,000 cycles was observed indicating that prototypes fabricated in this study were electrochemically stable. The long-term stability of eletroactive thin-film electrode material is the most substantial criterion for electrochemical capacitor application.

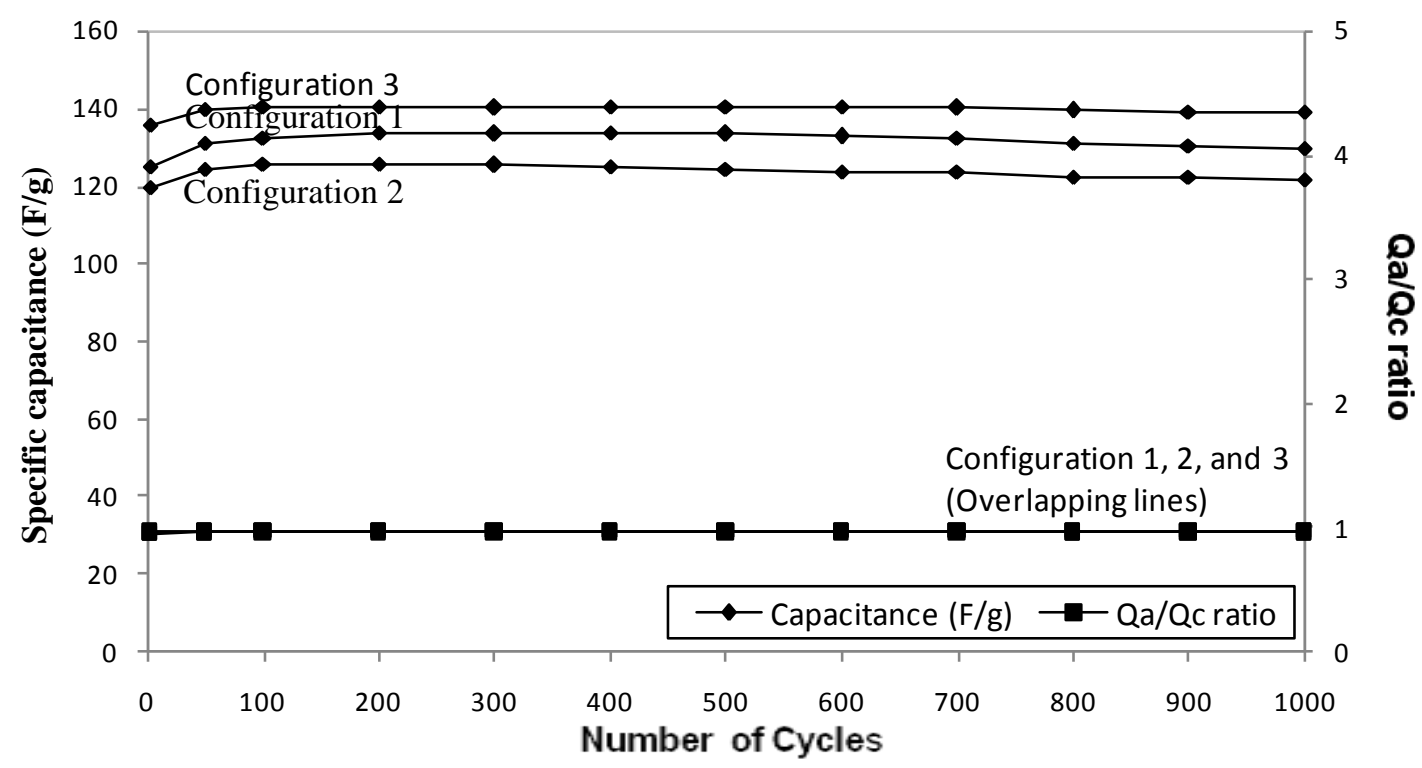

Figure 6. Cycling behaviors of electrochemical capacitor prototypes with different electrode configurations.

The observed excellent cycling stability of the $\mathrm{MnO}_{2}$ thin films for exceeding 1,000 cycles showed that there was no occurrence of structural modification and dissolution of active material throughout the prolong charge and discharge cycling test. We speculate that such high cycling stability and reversibility could be attributed to the amorphous nature of these films as well as the optimal scan potential window within the range of $-0.9 \mathrm{~V}$ to $0.9 \mathrm{~V}$ which had collectively prevented over-charging or over-discharging the prototype devices.

The specific capacitance of $\mathrm{MnO}_{2}$ thin films is believed to be predominantly contributed by pseudocapacitive behavior as a result of reversible redox reaction which corresponds to the reduction of $\mathrm{MnO}_{2}$. Double layer capacitance might have inconsequential effect upon the overall capacitance of these prototypes and was therefore neglected in this study. According to Wen et al., (2004), the charge storage mechanism of $\mathrm{MnO}_{2}$ involves rapid and reversible faradaic reaction through the cationic and protonic exchange. The redox processes for both $\mathrm{Na}^{+}$and $\mathrm{H}^{+}$ions which are present in the electrolytes could be shown in equations (3) and (4):

$$
\begin{aligned}
& \mathrm{MnO}_{2}+\delta \mathrm{Na}^{+}+\delta \mathrm{e}^{-} \rightleftharpoons \mathrm{MnO}_{2-\delta}(\mathrm{ONa})_{\delta} \\
& \mathrm{MnO}_{2}+\delta \mathrm{H}^{+}+\delta \mathrm{e}^{-} \rightleftharpoons \mathrm{MnO}_{2-\delta}(\mathrm{OH})_{\delta}
\end{aligned}
$$


The pseudocapacitance of a redox electrochemical capacitor might have been due to the amorphous nature of its electroactive material that constitutes the electrodes (Lee et $a l ., 1999)$. This could plausibly explain that the capacitance of $\mathrm{MnO}_{2}$ thin films prepared in the present study was attributed to its amorphous nature and porosity which collectively enabled high accessibility of $\mathrm{Na}^{+}$ions into the internal surfaces of these thin films. It is noteworthy to emphasize that factors which could significantly influence the rate of charging/discharging process of $\mathrm{MnO}_{2}$ and consequently the capacitance of the electrochemical capacitors include the size of both the cations and the hydration sphere of the cations in the electrolyte, the concentration of cations, the mobility of cations, the rate of adsorption/desorption of cations at the electrode surface and the pore size of the electroactive material (Wen et al., 2004). Lee et al. (1999) also reported that the pseudocapacitative performance of both electroactive materials, $\mathrm{K}_{\mathrm{x}} \mathrm{MnO}_{2+\delta} \cdot \mathrm{nH}_{2} \mathrm{O}$ and amorphous $\mathrm{MnO}_{2}$ was attributed to the $\mathrm{K}^{+}$ions which served as the working ions in complementary to the chemisorption and desorption of protons.

Considering the charge-storage mechanism that involves a total reduction of $\mathrm{Mn}^{4+}$ to $\mathrm{Mn}^{3+}$, such a redox reaction would contribute a theoretical specific capacitance of about 1,200 $\mathrm{F} / \mathrm{g}$. This specific capacitance value is approximately one order of magnitude larger than those values obtained in our prototype devices. However, this theoretical value has never been attained thus far and the average specific capacitance reported for $\mathrm{MnO}_{2}$ electrode tested in aqueous electrolyte is merely $160 \mathrm{~F} / \mathrm{g}$ (Brousse et al., 2006). Specific capacitance values achieved for our electrochemical capacitor prototypes were well within the same order of magnitude as the reported average value. Among parameters which might have significant influences upon the capacitive performance of the electrochemical capacitors include electrode design, type of electrolyte used, current collector, water content in the electrode material, pore size distribution, microstructure of $\mathrm{MnO}_{2}$, and $\mathrm{Mn}(\mathrm{III}) / \mathrm{Mn}(\mathrm{IV})$ ratio in the starting material.

\section{Electrochemical impedance spectroscopy (EIS)}

Figure 7 and Table 1 show the typical layout and dimensions of supercapacitor prototypes with three different electrode configurations. Configuration 1 comprised an identical pair of electrodes positioned horizontally with an interelectrode gap distance of $0.1 \mathrm{~mm}$. Both EC prototypes of configuration 2 and 3 comprise interdigitated array (IDA) electrodes of different electrode array lengths and widths. These prototypes of dual-planar thin-film electrochemical capacitors on flexible metalized PET substrate were fabricated using a novel cost-effective rapid prototyping process according to the desired electrode configurations. In this study, functional supercapacitor prototypes were fabricated with either $0.2 \mathrm{M} \mathrm{Na}_{2} \mathrm{SO}_{4}$ aqueous electrolyte or by depositing a thin layer of agar-based gel electrolyte containing $0.2 \mathrm{M} \mathrm{Na}_{2} \mathrm{SO}_{4}$ solution directly onto the $\mathrm{MnO}_{2}$ thin-film electrodes. IDA electrode configuration offers many pronounced advantages compared to the typical parallel electrodes which include rapid attainment of steady-state current, higher current collection efficiency, rapid redox reaction kinetic, higher detection sensitivity and selectivity, smaller charging current and smaller potential (iR) drop. 


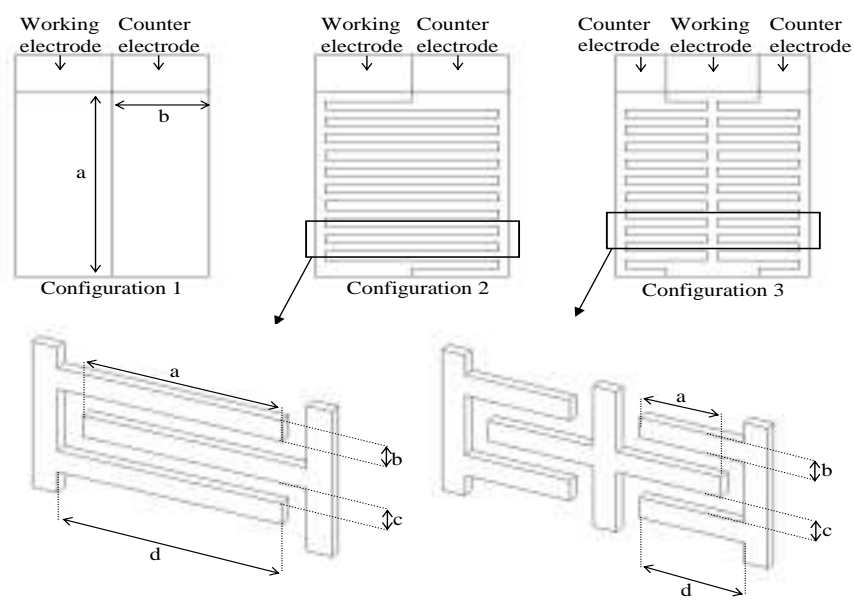

Figure 7. Layout of electrochemical capacitor prototypes of three different electrode configurations. (a) length of array electrode pair; (b) array electrode width; (c) interelectrode gap distance, and (d) length of individual array electrode.

The Nyquist plots for electrochemical capacitor prototypes of different electrode configurations being evaluated in different electrolyte media (liquid or agar-based gel electrolytes) are shown in Figures 8(a) - (h). EIS is a consequential and vastly applicable electrochemical characterization technique for any electrochemical cells, particularly electrochemical capacitors. The impedance plot for an ideal capacitor is a vertical line parallel to the imaginary impedance axis. Impedance plots which showed a steep slope in the low frequency region and a semicircular curve in the high frequency region were indicative of capacitive behaviors of typical electrochemical capacitors. Based on the Nyquist plots for all electrochemical capacitor prototypes, several types of resistances that could be identified at the high frequency range include contact resistance $R_{c}$ at the $\mathrm{MnO}_{2}$ active material/nickel current collector interface, electrolyte resistance $R_{s}$, and $\mathrm{MnO}_{2}$ electrode resistance $R_{e}$. The capacitance $C$ of the electrochemical capacitor prototypes could be derived from the low frequency regions of $100 \mathrm{mHz}$ and $10 \mathrm{mHz}$ using equation (2).

Table 1. Typical layout dimension for electrochemical capacitor prototypes of various electrode configurations.

\begin{tabular}{ccccc}
\hline Prototype & \multicolumn{4}{c}{ Dimension $(\mathbf{m m})$} \\
\cline { 2 - 5 } Configuration & $\mathbf{a}$ & $\mathbf{b}$ & $\mathbf{c}$ & $\mathbf{d}$ \\
\hline $\mathrm{C} 1$ & 16.0 & 5.0 & 0.1 & N/A \\
$\mathrm{C} 2$ & 7.8 & 1.0 & 0.1 & 7.9 \\
$\mathrm{C} 3$ & 3.3 & 1.0 & 0.1 & 3.4 \\
\hline
\end{tabular}

Key: "N/A" indicates not applicable. 
As shown in Figure 8, all electrochemical capacitor prototypes exhibited typical semicircular and steep vertical impedance responses in the high and low frequency regions, respectively. Prototypes with liquid electrolyte $\left(0.2 \mathrm{M} \mathrm{Na}_{2} \mathrm{SO}_{4}\right)$ and agar-based gel impedance plots for prototypes with agar-based gel electrolyte were observed to have shifted slightly to higher real impedance $Z_{\text {real }}$ as compared to that of liquid electrolyte. Figures 9(d) - (f), showed distinctive shift of the impedance plots particularly in the lower frequency regions for agar-based gel electrolyte prototypes before and after voltammetric cycling test. In addition, the semicircular impedance responses at higher frequency regions after cycling test were not as pronounced as those before long-term cycling test. These responses could be attributed to microstructural changes of electroactive material which ameliorates penetration of electrolyte into the recess area of the electrode materials. Figures $8(\mathrm{~g})$ and $(\mathrm{h})$ shows the Nyquist plots of prototypes in liquid electrolyte before long-term cycling test and prototypes in agar-based gel electrolyte after long-term cycling test, respectively. Both insets clearly showed the substantially diminished semicircular impedance plots for prototypes in agar-based gel electrolyte.

Polymer gel electrolyte constituting of polysaccharide such as agar gel containing dissolved salt could indeed replace liquid-based electrolyte in the fabrication of all-solid-state

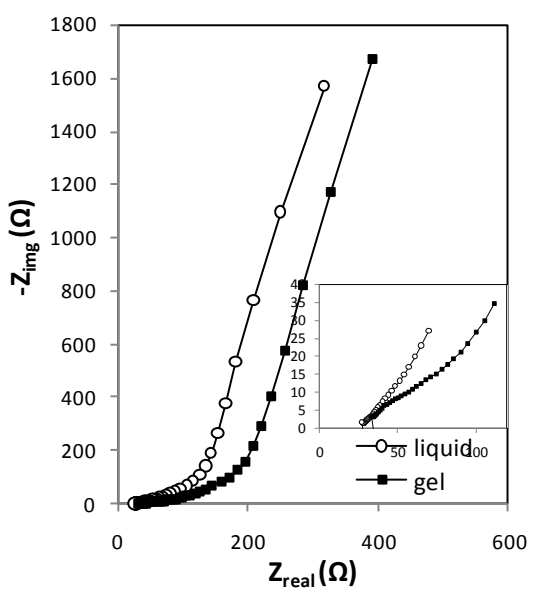

(a) electrochemical capacitor (Mochizuki et al., 2004). Results of EIS analysis have shown that agar-based polymer gel electrolyte $(1 \% \mathrm{w} / \mathrm{v})$ with dissolved $\mathrm{Na}_{2} \mathrm{SO}_{4}$ salt exhibited comparable ionic conductivity with that of 0.2 $\mathrm{M} \mathrm{Na} \mathrm{SO}_{4}$ liquid electrolyte. This could be attributed to similarity in the molecular diffusivity for both polysaccharide-based gel electrolyte and liquid electrolyte. In order for polymer gel electrolyte to perform equally well as liquid electrolyte, we speculate that the amount of electrolyte being encapsulated within pores of polymer gel electrolyte should be taken into account. It has been established that the amount of water or electrolyte plays a pivotal role in enhancing cationic intercalation into the bulk of the electroactive material electrolyte containing dissolved salt $(0.2 \mathrm{M}$ $\mathrm{Na}_{2} \mathrm{SO}_{4}$ ) did not show any distinctive differences in the electrolyte resistance $R_{s}$ at high frequency regions (Figure 9(a)-(c) insets).

However, based on the EIS data, the highest specific capacitance calculated at the lower frequency range for prototypes of configurations $\mathrm{C} 1, \mathrm{C} 2$, and $\mathrm{C} 3$ and packaged separately with gel electrolyte were determined to be $133 \mathrm{~F} / \mathrm{g}, 125 \mathrm{~F} / \mathrm{g}$, and $152 \mathrm{~F} / \mathrm{g}$, respectively. Specific capacitance values for electrochemical capacitor prototypes constructed with gel electrolyte were observed to be generally higher by about 15 to $30 \mathrm{~F} / \mathrm{g}$ after long-term cycling test. These results were observed to agree closely with specific capacitance values obtained by the cyclic voltammetry technique.

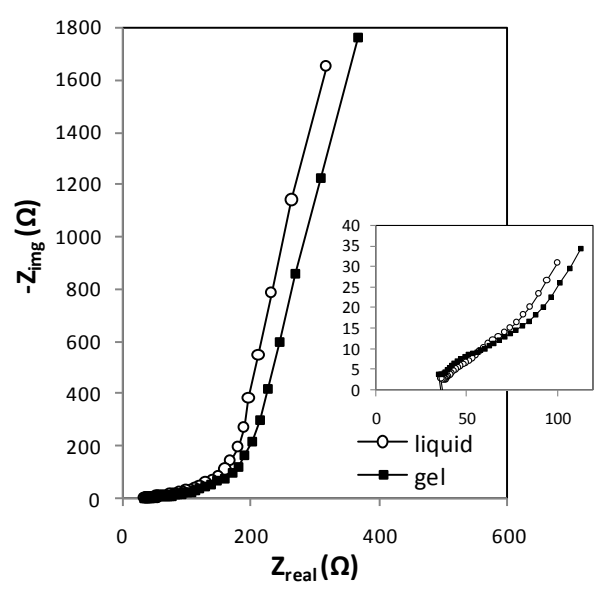

(b) 


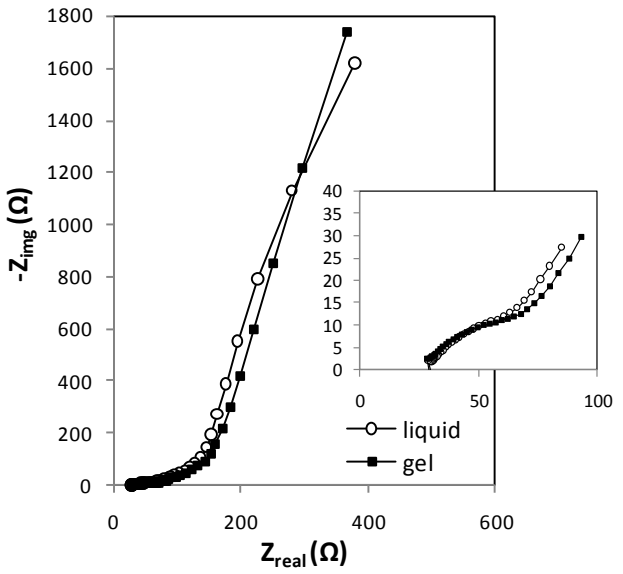

(c)

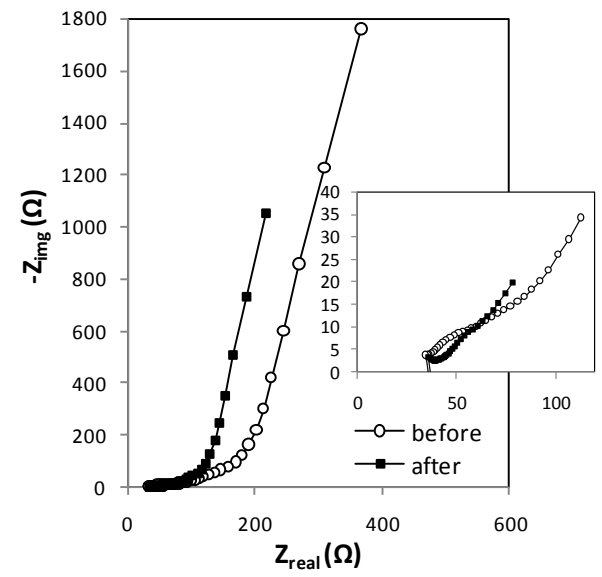

(e)

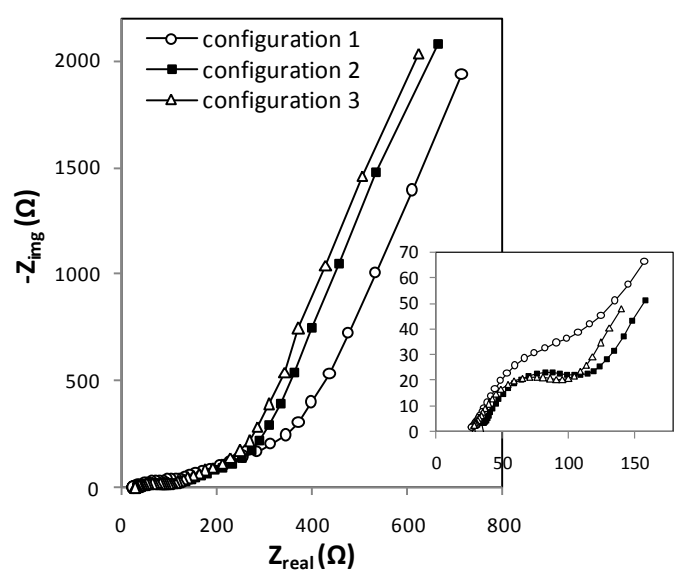

$(\mathrm{g})$

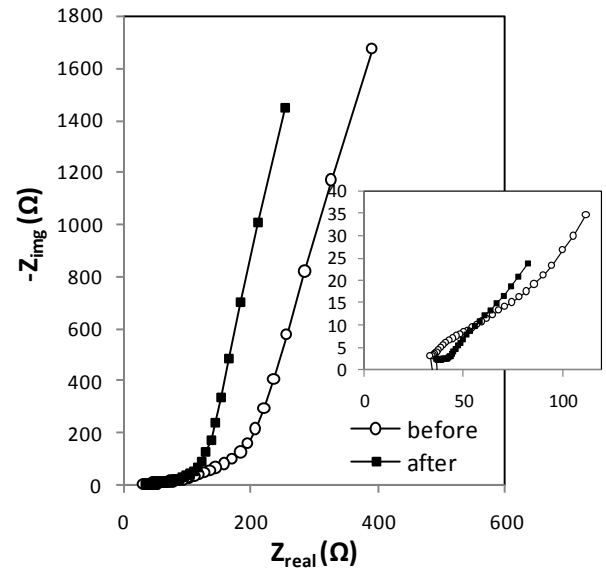

(d)

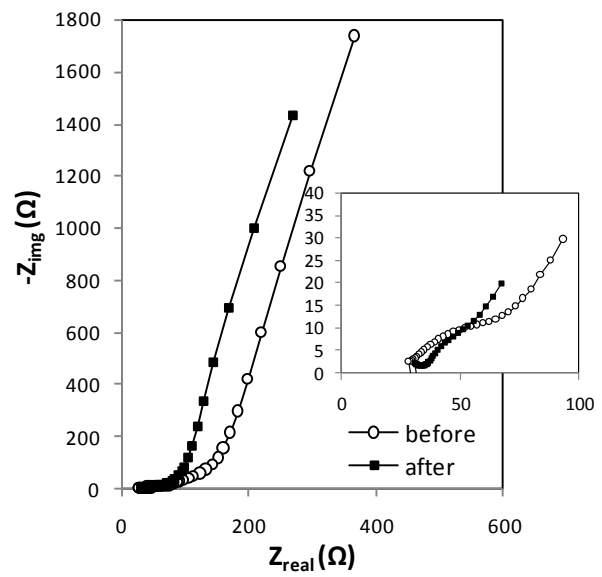

(f)

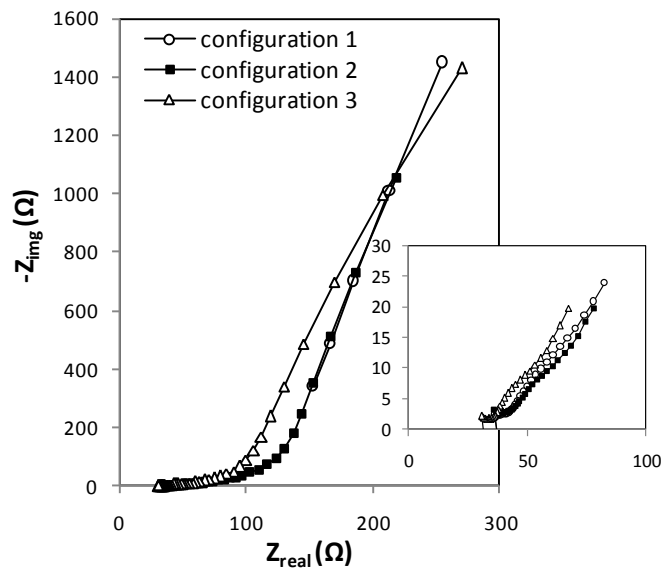

(h)

Figure 8. Nyquist plots of various electrochemical capacitor prototypes, (a) $\mathrm{C} 1$, liquid and gel, (b) $\mathrm{C} 2$, liquid and gel, (c) C3, liquid and gel, (d) C1, gel, before \& after cycling, (e) C2, gel, before \& after cycling, (f) C3, gel, before \& after cycling, (g) C1,2,3, liquid, before cycling, and (h) C1,2,3, gel, after cycling. All prototypes were tested at ambient temperature and $100 \% \mathrm{RH}$ within the frequency range of $1 \mathrm{MHz}$ to $10 \mathrm{mHz}$. C: configuration; Electrolyte: $\mathrm{Na}_{2} \mathrm{SO}_{4}$ (liquid) electrolyte; Agar-based (gel) electrolyte. 


\section{CONCLUSION}

Nanostructured manganese dioxide thin films prepared by a novel self-assembly process were nanoparticulate and highly porous in nature. The microstructure and electrochemical properties of these self-assembled films could be tailored through optimization of the deposition parameters and conditions. Selfassembled manganese dioxide thin films exhibited excellent capacitive behavior with high cycling stability and reversibility for exceeding 1,000 charge-discharge cycles. As such, self-assembled manganese dioxide thin films are very promising electrode materials for the fabrication of high power and energy density thin-film electrochemical capacitors.

\section{ACKNOWLEDGEMENTS}

This work was jointly supported by the Ministry of Higher Education (MOHE) Fundamental Research Grant Scheme (FRGS/01(18)/747/2010(33) and Ministry of Science, Technology and Innovation (MOSTI) E-Science Fund (03-01-09SF0076)

\section{REFERENCES}

Brousse, T., Toupin, M., Dugas, R., Athouel. L., Crosnier, O., \& Bèlanger, D. (2006). Crystalline $\mathrm{MnO}_{2}$ as Possible Alternatives to Amorphous Compounds in Electrochemical Supercapacitors. Journal of the Electrochemical Society, 153 (12): A2171 - A2180.

Chin, S. F. Pang S. C., \& Anderson M.A. (2002). Material and Electrochemical Characterization of Tetrapropylammonium Manganese Oxide Thin Film as Novel Electrode Materials for Electrochemical Capacitors. Journal of Electrochemical Society, 149(4): A379 - 384.

Dai, Y., Wang, K., Zhao, J. C., \& Xie, J. Y. (2006). Manganese oxide film electrodes prepared by electrostatic spray deposition for electrochemical capacitors from the $\mathrm{KMnO}_{4}$ solution. Journal of Power Sources, 161: 737 - 742 .
Lee, H. Y., Manivannan, V., \& Goodenough, J. B. (1999). Electrochemical capacitors with $\mathrm{KCl}$ electrolyte. Comptes Rendus de l'Académie des Sciences - Series IIC Chemistry, $565-577$.

Liu, E. H., Meng, X. Y., Ding, R., Zhou, J. C., \& Tan, S. T. (2007). Potentiodynamical codeposited manganese oxide/carbon composite for high capacitance electrochemical capacitors. Materials Letters, 61: 3486 - 3489.

Mochizuki, N., Ueno, H., \& Kaneko, M. (2004). Solid medium for conventional electrochemical measurements. Electrochimica Acta, 49: 4143 - 4148.

Nagarajan, N., Humadi, H., \& Zhitomirsky, I. (2006). Cathodic electrodeposition of $\mathrm{MnO}_{\mathrm{x}}$ films for electrochemical supercapacitors. Electrochimica Acta, 51: $3039-3045$.

Qu, D. Y. (2006). Investigation of the porosity of electrolytic manganese dioxide and its performance as alkaline cathode material. Journal of Power Sources, 156: 692 - 699.

Pang S. C., Anderson, M. A. \& Chapman, T. W. (2000) Novel Electrode Materials for Thin-Film Ultracapacitors: I. Comparison of Electrochemical Properties of Sol-GelDerived and Electrodeposited Manganese Dioxide. Journal of Electrochemical Society, 147(2): 444-450.

Perez-Benito, J. F., Arias, C. \& Amat, E. (1996). A kinetic study of the reduction of colloidal manganese dioxide by oxalic acid. Journal of Colloid and Interface Science, 177: 288-297.

Wen S., Lee, J. W., Yeo, I. H., Park, J. M., \& Mho, S. I. (2004). The role of cations of the electrolyte for the pseudocapacitive behavior of metal oxide electrodes, $\mathrm{MnO}_{2}$ and $\mathrm{RuO}_{2}$. Electrochimica Acta, 50: 849 855. 
Yagi, H., Ichikawa, T., Hirano, A., Imanishi, N., Ogawa, S., \& Takeda, Y. (2002). Electrode characteristics of manganese oxides prepared by reduction method. Solid State Ionics, 154 -155: 273 - 278.
Zolfaghari, A., Ataherian, F., Ghaemi, M., \& Gholami, A. (2007). Capacitive behavior of nanostructured $\mathrm{MnO}_{2}$ prepared by sonochemistry method. Electrochimica Acta, 52: 2806 - 2814. 